\title{
Defining Tinkering Behavior in Open-ended Block-based Programming Assignments
}

\author{
Yihuan Dong, Samiha Marwan, Veronica Cateté, Thomas Price, Tiffany Barnes \\ North Carolina State University \\ Raleigh, North Carolina \\ \{ydong2,samarwan,vmcatete,twprice,tmbarnes\}@ncsu.edu
}

\begin{abstract}
Tinkering has been shown to have a positive influence on students in open-ended making activities. Open-ended programming assignments in block-based programming resemble making activities in that both of them encourage students to tinker with tools to create their own solutions to achieve a goal. However, previous studies of tinkering in programming discussed tinkering as a broad, ambiguous term, and investigated only self-reported data. To our knowledge, no research has studied student tinkering behaviors while solving problems in block-based programming environments. In this position paper, we propose a definition for tinkering in block-based programming environments as a kind of behavior that students exhibit when testing, exploring, and struggling during problem-solving. We introduce three general categories of tinkering behaviors (test-based, prototype-based, and construction-based tinkering) derived from student data, and use case studies to demonstrate how students exhibited these behaviors in problem-solving. We created the definitions using a mixed-methods research design combining a literature review with data-driven insights from submissions of two open-ended programming assignments in iSnap, a block-based programming environment. We discuss the implication of each type of tinkering behavior for learning. Our study and results are the first in this domain to define tinkering based on student behaviors in a block-based programming environment.
\end{abstract}

\section{CCS CONCEPTS}

- Social and professional topics $\rightarrow$ Computing education; • Applied computing $\rightarrow$ Interactive learning environments;

\section{KEYWORDS}

Tinkering; programming; novice; block-based

\section{ACM Reference Format:}

Yihuan Dong, Samiha Marwan, Veronica Cateté, Thomas Price, Tiffany Barnes. 2019. Defining Tinkering Behavior in Open-ended Block-based Programming Assignments. In Proceedings of the 50th ACM Technical Symposium on Computer Science Education (SIGCSE '19), February 27-March 2, 2019, Minneapolis, MN, USA. ACM, New York, NY, USA, 7 pages. https: //doi.org/10.1145/3287324.3287437

Permission to make digital or hard copies of all or part of this work for personal or classroom use is granted without fee provided that copies are not made or distributed for profit or commercial advantage and that copies bear this notice and the full citation on the first page. Copyrights for components of this work owned by others than ACM must be honored. Abstracting with credit is permitted. To copy otherwise, or republish to post on servers or to redistribute to lists, requires prior specific permission and/or a fee. Request permissions from permissions@acm.org.

SIGCSE '19, February 27-March 2, 2019, Minneapolis, MN, USA

(C) 2019 Association for Computing Machinery.

ACM ISBN 978-1-4503-5890-3/19/02 .\$15.00

https://doi.org/10.1145/3287324.3287437

\section{INTRODUCTION}

Block-based programming environments are specifically designed to help novices tinker and explore to learn programming. By using blocks over text-based languages, these environments reduce the learner's cognitive demands by limiting possible syntactic and grammatical mistakes and focusing only on developing algorithmic thinking [2, 8]. Additionally, block-based programming environments has been shown to increase learning and programming efficiency [16, 24]. Together with other known benefits [23], these attributes make block-based programming ideal in introductory programming courses [24], especially those targeted for novices.

When teaching with block-based programming, open-ended creative assignments are often used as a way to motivate students to learn. Block-based programming environments such as Scratch and Snap! are designed to encourage learners to explore the blocks and discover different ways of solving problems through tinkering [18]. Tinkering has been proven to have a positive effect on the makers in terms of their ability to express new realizations and offer explanations for strategies and tools [21]. Tinkering embraces creativity and gives learners the opportunity to explore and learn with hands-on experience. Open-ended programming assignments in block-based programming environments resemble making activities in that both of them encourage students to tinker with tools, virtually or physically, and create a solution to a problem. Thus, it is intuitive to assume that tinkering will help learners progress while problem-solving. However, to our knowledge, no previous research has defined what tinkering is in block-based programming, or studied the tinkering behaviors the learners exhibit in openended programming assignments, which may provide insights for the design and use of tinkering-focused interventions for learning.

We seek to better understand tinkering behaviors, and to eventually build automated systems to detect and support productive tinkering. In this work, we focus on the following two questions:

(1) What is tinkering behavior in open-ended block-based programming assignments?

(2) What are the different types of tinkering behaviors among novices during problem-solving?

In this position paper, we first discuss related work on tinkering and introduce Snap!, a block-based programming environment. Afterwards, we describe the methodology we used to analyze exploratory tinkering behaviors in two assignments from a nonmajors introduction to computers course. Then, we propose three types of tinkering that we observed in the student block programming trace data, illustrating these types with case studies. Finally, we discuss the purpose of identifying tinkering and its potential to benefit programming education. This work takes the first step 
in defining tinkering in block-based programming environments, especially for open-ended programming activities.

\section{BACKGROUND}

\subsection{Tinkering}

In recent decades, researchers have investigated methods and techniques that increase learning motivation and capability of exploration. In return, these techniques will increase the capability of learning and performing tasks effectively [7, 22]. As a result, the term tinkering emerges from makerspace activities. Some researchers considered tinkering as a "mindset" that involves a playful approach to solving problems through "direct experience, experimentation, and discovery" [10], which the mindset can cut across intentional building and disciplinary construction activities [21]. Other researchers considered tinkering as a type of making activities, instead of mindset $[14,19]$. However, none of the previous tinkering research provide actionable definition to identify tinkering behaviors in the making activities.

A number of studies have assessed the efficacy of tinkering behavior in improving the motivation in learning and exploring in different fields, like making, engineering, robotics and computer programming $[3,9,21]$. In addition to these studies, some previous tinkering research focused on analyzing tinkering as a way to explore gender differences in approaches to problem solving $[1,6]$. The Coursera course "Tinkering Fundamentals, A Constructionist Approach to STEM Learning" argues for the use of tinkering by educators and learners to develop their learning [13]. In [1], authors introduced tinkering as a behavior similar to debugging, as a trialand-error process.

The first broad definition of tinkering in computer science was introduced by Krieger et al [6]. Authors categorized tinkering behavior used by undergraduate students in computer science into four categories, named: Exploratory behaviors, Deviation from instructions, Lack of reliance on formal methods of learning and instruction, and Use of trial and error techniques. Authors used these categories to investigate the differences in tinkering between genders. Although the authors provide tinkering categorizations, their data is collected from surveys and questionnaires about previous programming assignments and not on actual student work during problem solving.

To our knowledge, there are no prior studies that provide a precise definition or categorization of tinkering based on student problem-solving data in block based programming environments.

\subsection{Block-based Programming Environments}

In this section, we first provide an overview of the Snap! blockbased programming environment and then give a brief discussion of the related literature illustrating how it has been specifically designed to promote tinkering. Scratch is a block-based environment designed to inspire children aged around 10-12 years old to explore programming by constructing creations of their own [18]. Snap! was inspired by Scratch, but was built specifically to support more advanced computer science and programming constructs, including custom blocks and recursion, for the "Beauty and Joy of Computing" version of the Computer Science Principles Advanced Placement course [5]. Figure 1 shows the interface of Snap!. The left palette holds the blocks, organized in 8 categories, that can be used to write a program. The user can drag and drop the blocks into the scripting area and snap the blocks together to make scripts. The user is able to create variables and custom blocks. The custom block resembles functions or methods in other programming languages and can be edited in the block editor. The stage is where the user can see the script outputs. The user can run a script either by clicking on the green flag to run the scripts attached to the "when green-flag clicked" block, or by clicking on an individual script. Below the stage is a Sprite palette that stores the sprites, which resembles the idea of an object in object-oriented programming languages. Each sprite can have its own behavior dictated by its variables and scripts and the user can switch between the sprites by clicking on the sprite icon.

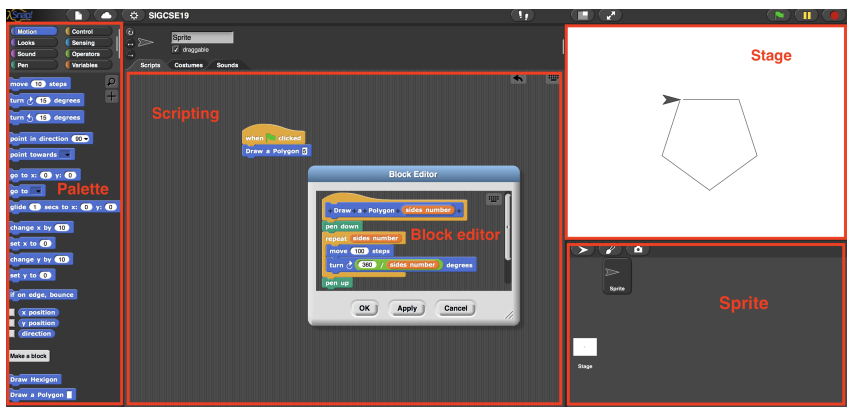

Figure 1: SNAP!, a Block-based Programming Environment.

Block-based programming environments are specifically designed to support a constructionist theory of learning [8]. They are considered easier to use than text-based alternatives [16, 24], helping prevent most syntactic errors [2]. The drag-and-drop interaction style mitigates the low proficiency of typing skills among young learners, and provides a visual way to learn the available commands. The natural language labels on the blocks make them easier to understand [8].

Moreover, block-based programming environments are designed to support construction, creativity, and learning through tinkering [20]. Blocks serve as virtual manipulatives like the mechanical and/or electronic objects in typical making activities. Blocks can be snapped together to make script snippets where both the blocks and the scripts can be tested by a single click. This sandbox-style, rapid feedback is meant to facilitate and encourage tinkering and construction. As such, block-based programming environments encourage users to tinker by creating and testing small features, then combining the features to reach a user-identified goal.

Although block-based programming environments are designed to support tinkering, there is no guarantee that the users are tinkering in a meaningful, productive way [20]. Studying the ways that students tinker may provide insights for designing curricula, interventions, and tools that improve student learning.

\section{METHOD}

In order to identify and categorize tinkering behavior, we looked at submissions of two programming assignments used in a non-majors "Introduction to Computers" course with 68 students. One section of 


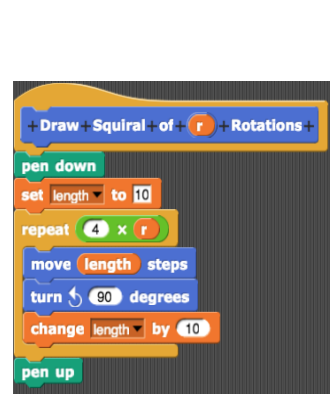

(a) Squiral Solution

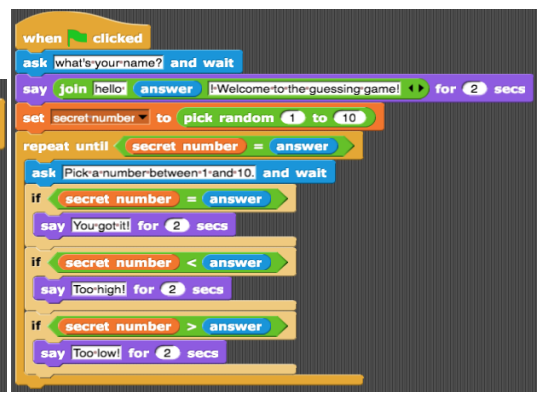

(b) Guessing Game Solution
Figure 2: Sample solutions for the Squiral assignment and the Guessing Game Assignment

the course uses labs from the BJC curriculum [5], including Squiral and Guessing game. Figure 2 shows the sample solutions for both the Squiral and Guessing game assignments. In the Squiral assignment, students make a custom block to draw a square-like spiral. The custom block needs to take a parameter that controls the number of full rotations for the Squiral. In the Guessing Game assignment, students create an interactive game where the player tries to guess a secret number. The game should start by asking and greeting the player by name, set a (random) secret number between 1 and 10, repetitively ask the player to guess the secret number until correct, tell the player if the player's guess was too high or too low, and then congratulate the player if the guess was correct.

The students programmed the assignments in iSnap, a variant of the Snap! programming environment that has the ability to provide students with next-step-hints upon student request [17]. iSnap captures a student's interactions inside the environment as a trace and is able to recreate the process of the student making the solution from the trace. Our analysis included student traces who requested next-step-hints during the assignments because the student needed to tinker to understand the hints and make progress. However, we filtered out 11 Squiral traces and 3 Guessing Game traces which completed the assignment by blindly requesting and following the next-step hints. The remaining dataset includes 46 Squiral traces and 62 Guessing Game traces.

Two experts, the first and second authors, took a mixed-methods research approach to define the observed tinkering behaviors. Both experts are proficient in programming in Snap! and one of them has extensive experience researching and analyzing student interactions in Snap!. The process of defining tinkering behaviors was conducted in 3 phases: initial definition, consensus, and verification, described as follows:

Phase 1 - Initial definition: The two experts started off by reviewing the literature on tinkering behaviors in makerspaces, programming, and similar activities, to get a general idea of how tinkering behavior is defined in previous research. Next, the two experts manually looked through the step-by-step interactions of all the traces on both assignments independently. Each expert created an initial definition of tinkering behaviors they observed in the interaction traces and categorized those behaviors. Then, the two experts discussed the tinkering behavior categorization they found in the dataset and merged their findings into one definition and categorization that they both agreed on.

Phase 2 - Consensus: The two experts then analyzed a shared set of 10 Squiral traces and 10 Guessing game traces. A random selection algorithm was used to select the 20 traces so that distribution of the samples across grade bins of 0-24, 25-50, 51-75, and 76-100 matched that of the total set of traces for each assignment. The experts took notes on the occurrences of each type of tinkering behavior on each of the 20 traces. Then, the experts discussed their notes to see if they agreed on all the occurences and types of tinkering, and resolved disagreements through discussion and updates to the definition and categorizations. This ensured that the experts had the same understanding of the definition and categories.

Phase 3 - Verification: Finally, the experts divided the remaining 88 traces, tagged the traces individually with the existing definitions, and made sure no tinkering behavior in the dataset was omitted from the definition and categorization. We note that different tinkering behaviors can occur at different times and for different reasons while students work on an assignment.

We present our results on defining tinkering behaviors by giving the definition of each type of tinkering behavior and illustrating the behavior through representative case studies. Purposive sampling is "based on the assumption that one wants to discover, understand, or gain insight; therefore one needs to select a sample from which one can learn the most" [12]. Therefore, the following case studies were selected using purposive sampling to demonstrate typical or interesting behaviors that can provide insights into each type of tinkering behavior.

\section{TINKERING BEHAVIOR IN BLOCK-BASED PROGRAMMING ASSIGNMENTS}

Our work focuses on defining tinkering behaviors in open-ended assignments using block-based programming environments. Openended programming assignments often provide students a set of goals to achieve, but do not restrict the students to have a single correct solution. These assignments, as opposed to step-by-step, prescriptive programming activities, give students more opportunities to freely explore the programming environment and embrace creative solutions to the given problem. As such, we expect students to display more tinkering behaviors in the process of completing open-ended programming assignments.

In this work, we define tinkering as a kind of behavior that students exhibit when testing, exploring, and struggling with their code to achieve a goal in a programming assignment. We consider a main theme of tinkering is a sense of uncertainty or hesitation within the student behavior, which can often be observed when a student moves a block back-and-forth or inserts and removes a block indecisively. We do not consider a student to be tinkering if the student knows exactly what to do and completes a feature or the whole assignment quickly and with minimal hesitation or revision. On the other hand, our definition's inclusion of working towards a goal requires tinkering to be purposeful. In this paper, the intention of the tinkering actions, whether tinkering with the "correct" blocks 
Table 1: Number of traces that exhibited each type of tinkering behavior in Phase 2 .

\begin{tabular}{|c|c|c|c|c|c|c|}
\hline & \multicolumn{2}{|l|}{ Test-based } & \multicolumn{2}{|l|}{ prototype-based } & $\begin{array}{c}\text { Const.- } \\
\text { based }\end{array}$ & $\begin{array}{c}\text { No } \\
\text { tinker }\end{array}$ \\
\cline { 2 - 6 } & debugging grunning & $\begin{array}{r}\text { 1nk- } \\
\text { sroject }\end{array}$ & expanding & & \\
\hline $\begin{array}{c}\text { Squiral } \\
\text { (n=10) }\end{array}$ & 6 & 8 & 4 & 3 & 6 & 2 \\
\hline $\begin{array}{c}\text { Guessing } \\
\text { Game } \\
(\mathrm{n}=10)\end{array}$ & 4 & 3 & 4 & 8 & 9 & 1 \\
\hline Total & 10 & 11 & 8 & 11 & 15 & 3 \\
\hline
\end{tabular}

used in the final solution or not, should be to achieve a goal or a subgoal in the assignment. Since programming assignments are usually designed with learning objectives in mind, we consider completely irrelevant, playful behaviors as more of an off-task behavior than a tinkering behavior, even though they can be considered as tinkering behaviors in some contexts [20]. Similarly, we excluded painting sprite costumes from our tinkering definition, because this type of tinkering is not related to learning programming.

We identified three general categories of tinkering behaviors in the two programming assignments: test-based tinkering, prototypebased tinkering, and construction-based tinkering. Test-based tinkering can be further categorized into debugging and running, and Prototype-based tinkering can be further categorized into sideproject and expanding. Table 1 shows the number of tinkering behaviors observed in the 20 traces analyzed in the consensus phase. The considerable number of examples suggests that all of these behaviors are prevalent in student programming traces, with only 3 of the 20 samples exhibiting no tinkering. We describe the detailed definition for each type of tinkering behavior in the following sections. We note that our categorization is not meant to be exhaustive. Instead of attempting to fit a tinkering behavior into a single category, we focused more on depicting the main characteristics present in each tinkering behavior we observed. Therefore some tinkering behaviors may fit into two or more categories, and each trace may contain many examples of tinkering.

\subsection{Test-based Tinkering}

Test-based tinkering is a type of behavior that involves executing the part of the code the student is editing. Students who exhibit test-based tinkering tend to test their scripts frequently within a few edits or in a short amount of time. Test-based tinkering can be further categorized into two sub-categories: debugging and running.

\section{Debugging}

Debugging refers to students who adopt trial-and-error behaviors when fixing a bug, defined as an unexpected or undesirable script output. Debugging is a well-known process where programmers

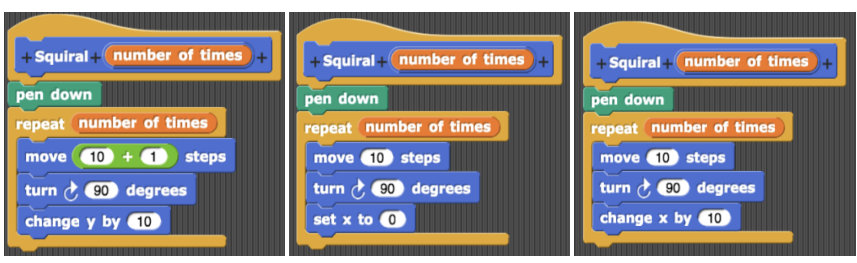

Figure 3: Debugging case study 1
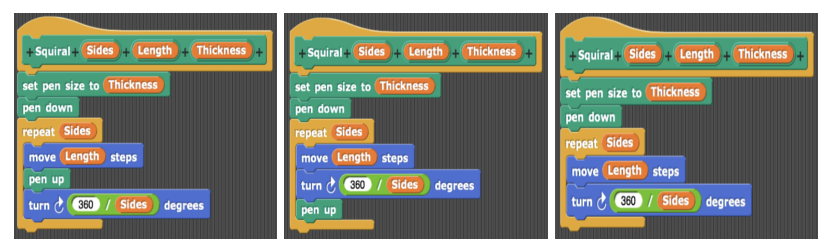

Figure 4: Debugging case study 2

make small changes to the code to locate and correct an error. In the context of block-based programming, students with this debugging behavior generally start with a script not working as intended, usually for an unknown reason. The student would make minor changes in the script followed by immediate testing in an attempt to fix the anomaly. Here, "minor changesâĂİ refers to changes that do not impact the overall structure of the code, for example, tweaking the parameters of blocks, reordering, and adding or removing blocks. This behavior can help students explore the functionality of the blocks and identify the reason for the differences between the desired and actual output. It is worth noting that novices tend to debug unconsciously without using a systematic approach as opposed to professionals.

In one case, a student, whom we will call Alex, got stuck trying to code correct blocks in the loop to draw a Squiral. Alex started to tinker with no purpose by using irrelevant blocks and testing the updated code afterwards. As shown in Figure 3, Alex first used the "change y" block, which is used to change the y-coordinate value and this is completely irrelevant to the activity. Afterwards, she tried using the "set $\mathrm{x}$ " block, which is used to set the $\mathrm{x}$-coordinate, afterwards she used the "change $\mathrm{x}$ " block and tested again and still did not get a desired output. It is relatively clear that the student's goal was to increase side length after each turn. Although this behavior did not help the student approach the solution, it might have helped rule out those blocks from the solution and explore those blocks' functionalities.

On the other hand, while solving the Squiral exercise, a student whom we call Tay, got stuck determining where to place the "PenUp" block, as shown in Figure 4. Tay started debugging and tinkering by placing the "PenUp" block in the middle of a loop, and then tested the code. Tay found that the sprite, instead of drawing a square, only draws a line. Next, Tay tried to place the "PenUp" block at the end of the loop then tested the script again. The output was, again, only drawing a line. At this point, Tay seemed to realize that the "PenUp" block does not belong inside the loop and finally removed the "PenUp" block from the script. This behavior clearly illustrates a trial and error manner that is useful in exploring the arrangement of the blocks. 


\section{Running}

Running describes a case when the student runs the code several times consecutively after some edits. The main difference between Running and Debugging is that Running involves multiple runs without making any code edits in between, while Debugging requires the student to make progress by regularly running the code with meaningful edits between the runs. Running behavior can be either helpful or harmful in different cases. On the one hand, Running behavior sometimes indicates the student is trying to interpret how the script works by looking at the output of each run. This usually happens when the code can execute without runtime errors but the output is undesirable. On the other hand, running behavior could be an indicator of frustration. For example, one student ran a script over 15 times, even though the script couldn't run properly because of using a variable named "Length" with a capital L instead of "length". The student was frustrated by not being able to figure out why the code did not work as expected. In this case, running behavior is considered an anti-pattern.

\subsection{Prototype-based Tinkering}

Prototype-based tinkering behavior is a way to complete a feature by first creating and testing a prototype of the feature. The prototype could be a part of a feature, with clear similarities to other parts, for example three consecutive if blocks to model three different conditions in a feature. The prototype could also be a feature assembled and tested aside from the main script, and later integrated or replicated in the main script. This behavior is similar to the bottom-up programming habits described in Meerbaum-Salant's research on novice programmer habits in text-based environments [11]. While Meerbaum-Salant considered the bottom-up programming habit to be a general problem-solving behavior, the prototype-based tinkering that we propose here is smaller in scope, e.g. completing a feature or even a part of a feature, and can appear alternately with other tinkering behaviors in student interactions. We categorized prototyping-based tinkering behaviors into two subcategories: side-project, and expanding.

\section{Side Project}

Side-project describes a behaviour where students build up code blocks (no matter the size) outside of an identifiable part of the main solution script. For example, a student tinkers with a set of blocks to assemble a small feature of the assignment. After testing its correctness or sometimes reasoning with logic, the student integrates the side-project into the main script. Another example is a student who builds up several small functionalities, each one on its own and then integrates them. Figure 5 presents an example where a student created a condition, tested it, and then added it to the main script. It is interesting that this student adopted this testing behavior multiple times throughout their work.

\section{Expanding}

Expanding describes a behaviour where the student shows a strong intention of prototyping and testing a part of a feature and then expands or just replicates it to develop the whole feature. The part of the feature usually is a representative chunk that appears multiple

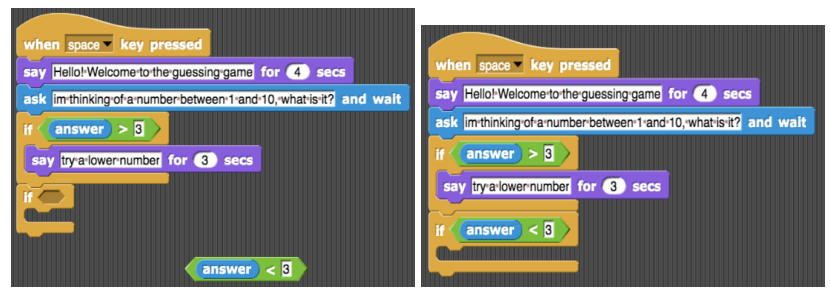

Figure 5: Side Project case study
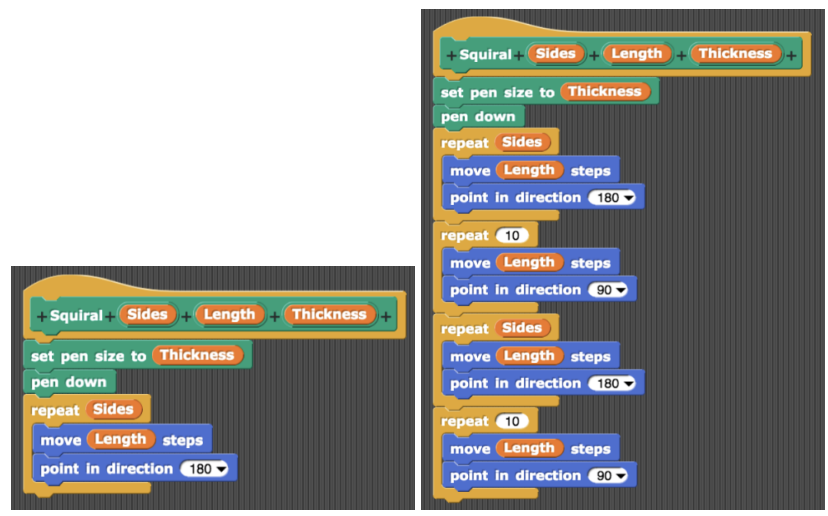

Figure 6: A Case Study on Unproductive Expanding
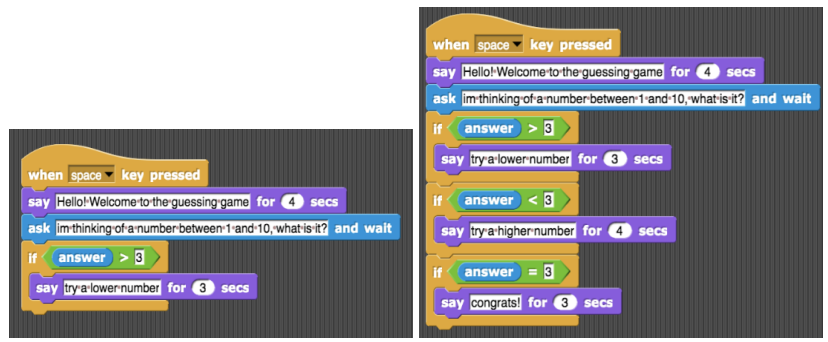

Figure 7: A Case Study on Productive Expanding

times in the feature with similar functionality. Expanding can be done both productively and unproductively, as illustrated by two cases below. Our first case study of Em represents an unproductive example of Expanding while solving the Squiral exercise, as shown in, Figure 6.. Em started a sequence of three blocks, then ran it, duplicated it and ran it again, and so on. Although this behavior reflects tinkering, we argue that it is not productive, since the use of blocks in this way misguides Em away from the correct use of the repeat pattern.

On the other hand, Figure 7 shows a case study of a student, Max, who adopts a productive Expanding behavior on the Guessing game. Max created an if-condition and made three copies of it to cover all the cases needed for the solution. Although the figure does not show the final solution, these three if conditions are needed to guarantee a correct final solution.

\subsection{Construction-based Tinkering}

Construction-based Tinkering is a behavior where students spend a good amount of time making major changes in the code, like 

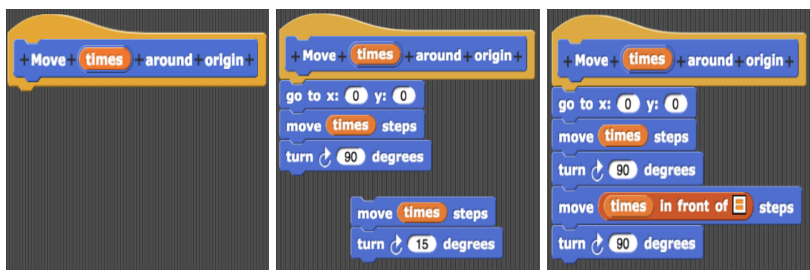

Figure 8: A Case Study on Construction based Tinkering

adding, deleting, or rearranging blocks, before any testing. Figure 8 shows an example of a student with Construction-based tinkering behavior, where the student constantly manipulated the blocks and inputs without testing. Construction-based tinkering behavior indicates that the student is likely pursuing an idea on how to solve the exercise, but still demonstrates some hesitation or uncertainty. We differentiate Construction-based tinkering from non-tinkering construction behavior based on the amount of hesitation or uncertainty exhibited. Construction-based tinkering involves pauses, or uncertainty in student actions, including major edits, or repeatedly adding and deleting large numbers of blocks. When students quickly construct large chunks of a solution with minimal hesitation, this indicates that the student has a prescribed idea on how to reach the solution without the need for exploration. Hence, we do not classify this expert-like construction behavior as tinkering.

Construction-based tinkering differs from debugging based on larger script changes with infrequent testing. Test-based tinkering is characterized by student tests after small edits, while constructionbased tinkerers test complete features all at once.

\section{DISCUSSION}

The purpose of identifying and categorizing tinkering behavior is not to say which tinkering behaviors are good or bad. In fact, any of the tinkering behaviors identified, depending on the student mindset, can turn out to be productive or detrimental. For example, with the test-based running behavior, a student could run a piece of script multiple times, attempting to understand how the script works by analyzing the output after each run. This is often encouraged and considered as a positive behavior. In other cases, students could be running the script numerous times, not trying to understand how the script works, but only as a sign of frustration.

Our purpose in studying tinkering behavior is to learn about students' behavior when approaching a problem, and to identify moments of productive struggle. Struggling with some perplexity, confusion, or doubt has been considered a necessary component of learning by both mathematicians and cognitive scientists. Struggle becomes productive when it leads to a process of restructuring a disequilibrium towards new understanding [15]. By identifying student tinkering behavior, we hope to find ways to encourage and support productive struggle that helps students gain understanding, and prevent unproductive struggle that may lead to fatigue, boredom, or frustration.

Studying students' tinkering behavior opens up new opportunities to support student learning. One goal for identifying tinkering behavior is to automatically identify and categorize student's behavior in real time or for a static summary. One example where this can be used is to improve hint algorithms. Much research has been done to provide hints to help students complete programming assignments. While most hint generation algorithms focus on how to bring the students closer to a correct solution, few consider how to select meaningful hints that meet a student's most important current need. Irrelevant hints may lead to student distrust and as a result students may decide not to use hint features even when struggling [17]. Identifying student tinkering behavior may not only help to pinpoint the location where the student is struggling, but it may also help to track student's subgoals. Consequently, this process will help filter out hints not related to a student's struggle before presenting them to the student. In addition, automated tinkering detection can be used in building a dashboard [4] for instructors so that they can see students' tinkering behaviors in the programming environment and provide interventions accordingly.

This research may help us discover tinkering behaviors that need for a different kind of help. For example, if a student is showing running behavior indicating frustration, it maybe appropriate to suggest a hint or in-person help from the instructors. However, if a student is exhibiting construction-based tinkering behavior for a while, it may be better to suggest the student to test the code first instead of suggesting a next-step hint.

Moreover, studying tinkering behavior could guide the design of curricula for block-based programming environments. Since tinkering has been shown to have a positive effect in "making" activities, it is reasonable to hypothesize that tinkering behavior, when used meaningfully, can have a positive effect on learning to program. If proven, block-based programming tutorials can be improved to show students how they can productively tinker to discover the functionality of the blocks on their own.

One limitation of this work is that it only analyzed 108 code traces from two programming assignments in a college-level, nonmajor course. Thus, the tinkering behaviors identified are by no means exhaustive. Additional tinkering behaviors may exist among different age groups (e.g. K-12 students) and in different types of programming assignments (e.g. making adventure games).

\section{CONCLUSIONS AND FUTURE WORK}

In this position paper, we proposed a goal-oriented definition for tinkering in order to study exploratory behaviors in block-based programming. We took a data-driven, mixed-methods approach to investigate tinkering in 108 sample novice programs. Based on our analysis of two assignments in a non-majors intro to computers course, we identified three categories of tinkering behaviors: test-based, prototype-based, and construction-based tinkering. Our study shows that tinkering behavior can be productive and unproductive depending on how the student tinker in different cases. Our findings do suggest ways to differentiate between productive and unproductive tinkering. We were able to classify tinkering behaviors using programming traces, suggesting that it may be feasible to realize our goal to build an automated system to detect, support, and promote productive tinkering.

\section{ACKNOWLEDGMENTS}

This material is based upon work supported by the National Science Foundation under grant number 1623470. 


\section{REFERENCES}

[1] Laura Beckwith, Cory Kissinger, Margaret Burnett, Susan Wiedenbeck, Joseph Lawrance, Alan Blackwell, and Curtis Cook. 2006. Tinkering and gender in end-user programmers' debugging. In Proceedings of the SIGCHI conference on Human Factors in computing systems. ACM, 231-240.

[2] Andrew Begel. 1996. LogoBlocks: A graphical programming language for inter acting with the world. Electrical Engineering and Computer Science Department, MIT, Boston, MA (1996), 62-64.

[3] Marina Umaschi Bers, Louise Flannery, Elizabeth R Kazakoff, and Amanda Sullivan. 2014. Computational thinking and tinkering: Exploration of an early childhood robotics curriculum. Computers \& Education 72 (2014), 145-157.

[4] Nicholas Diana, Michael Eagle, John Stamper, Shuchi Grover, Marie Bienkowski, and Satabdi Basu. 2017. An instructor dashboard for real-time analytics in interactive programming assignments. In Proceedings of the Seventh International Learning Analytics \& Knowledge Conference. ACM, 272-279.

[5] Dan Garcia, Brian Harvey, and Tiffany Barnes. 2015. The beauty and joy of computing. ACM Inroads 6, 4 (2015), 71-79.

[6] Samantha Krieger, Meghan Allen, and Catherine Rawn. 2015. Are females disinclined to tinker in computer science? In Proceedings of the 46th ACM Technical Symposium on Computer Science Education. ACM, 102-107.

[7] Mark Lepper. 1987. Intrinsic motivation and instructional effectiveness in computer-based education. Aptitude, learning, and instruction: III. Conative and affective process analyses (1987), 255-296.

[8] John Maloney, Mitchel Resnick, Natalie Rusk, Brian Silverman, and Evelyn Eastmond. 2010. The scratch programming language and environment. ACM Transactions on Computing Education (TOCE) 10, 4 (2010), 16.

[9] Richard Martin and Andrew Klein. 2017. Improved student independence through competitive tinkering. In Frontiers in Education Conference (FIE). IEEE, 1-8.

[10] Sylvia Libow Martinez and Gary Stager. 2013. Invent to learn: Making, tinkering, and engineering in the classroom. Constructing modern knowledge press Torrance, CA.

[11] Orni Meerbaum-Salant, Michal Armoni, and Mordechai Ben-Ari. 2011. Habits of programming in scratch. In Proceedings of the 16th annual joint conference on Innovation and technology in computer science education. ACM, 168-172.

[12] Sharan B Merriam. 1988. Case study research in education: A qualitative approach Jossey-Bass.
[13] Mike Petrich, Karen Wilkinson, and Luigi Anzivino. 2014. Tinkering Fundamentals: A Constructionist Approach to STEM Learning. Coursera. (August 2014). www.coursera.org/course/tinkering

[14] Mike Petrich, Karen Wilkinson, and Bronwyn Bevan. 2013. It looks like fun, but are they learning. Design, make, play: Growing the next generation of STEM innovators (2013), 50-70.

[15] Jean Piaget. 2005. The psychology of intelligence. Routledge.

[16] Thomas W Price and Tiffany Barnes. 2015. Comparing textual and block interfaces in a novice programming environment. In Proceedings of the eleventh annual International Conference on International Computing Education Research. ACM, 91-99.

[17] Thomas W Price, Yihuan Dong, and Dragan Lipovac. 2017. iSnap: towards intelligent tutoring in novice programming environments. In Proceedings of the 2017 ACM SIGCSE Technical Symposium on Computer Science Education. ACM, 483-488.

[18] Mitchel Resnick, John Maloney, Andrés Monroy-Hernández, Natalie Rusk, Evelyn Eastmond, Karen Brennan, Amon Millner, Eric Rosenbaum, Jay Silver, Brian Silverman, et al. 2009. Scratch: programming for all. Commun. ACM 52, 11 (2009), 60-67.

[19] Mitchel Resnick and Eric Rosenbaum. 2013. Designing for tinkerability. Design, make, play: Growing the next generation of STEM innovators (2013), 163-181.

[20] Ian Utting, Stephen Cooper, Michael Kölling, John Maloney, and Mitchel Resnick. 2010. Alice, greenfoot, and scratch-a discussion. ACM Transactions on Computing Education (TOCE) 10, 4 (2010), 17.

[21] Shirin Vossoughi and Bronwyn Bevan. 2014. Making and tinkering: A review of the literature. National Research Council Committee on Out of School Time STEM (2014), 1-55.

[22] Jane Webster and Joseph J Martocchio. 1993. Turning work into play: Implications for microcomputer software training. Fournal of Management 19, 1 (1993), 127146.

[23] David Weintrop and Uri Wilensky. 2015. To block or not to block, that is the question: students' perceptions of blocks-based programming. In Proceedings of the 14th International Conference on Interaction Design and Children. ACM, 199-208.

[24] David Weintrop and Uri Wilensky. 2017. Comparing block-based and text-based programming in high school computer science classrooms. ACM Transactions on Computing Education (TOCE) 18, 1 (2017), 3. 